\title{
WEB-BASED APPLICATION FOR INTERACTIVE SELECTION OF IMAGE PROMINENT COLOURS
}

\author{
Primož Weingerl (D), Dejana Javoršek \\ University of Ljubljana, Faculty of Natural Sciences and Engineering, \\ Department of Textiles, Graphic Arts and Design, Ljubljana, Slovenia
}

\begin{abstract}
An image can contain myriad different colours, but only a few of them are noticeable at first sight, hence in some way are defining the image. Therefore, the question arises which colours are prominent and what are the main factors that affect their prominence. In this paper, we present a webbased application that supports an interactive selection of colours and can be used to gather prominent colours for a set of images. The resulted database of images and their corresponding colours can be further used for investigating which image features or colour properties contribute to their prominence and for validating the models for automatic extraction of prominent colours from the images. First, the perception of prominent colours is addressed, and applied perspective of this knowledge is given. In the remainder of the paper, an overview of application architecture is presented, and a more detailed description of application's settings and usage is given. Although the main purpose of our application is to gather prominent colours of images based on the observer's opinion, the application could also be used for conducting other psychophysical experiments. The application supports three different modes for selecting the prominent colours: selection of basic hues, selection from ColorChecker Classic target and selection from custom defined patches.
\end{abstract}

Key words: web application, colour scheme, prominent colours, colour selection

\section{INTRODUCTION}

Colour is a fundamental sensation, which helps us to interpret and understand the material world that surrounds us. It affects our emotions and visual attention (Ou et al, 2004; Kopacz, 2012), so we could use it to deliver the desired information to the end user. Consequently, image colours can convey different emotions (Liu et al, 2018) and therefore influence the information value of the image. However, from the technical aspect, an image can contain myriad different colours. This raises an interesting question of which colours are most prominent - noticeable at first sight, and what are the main factors that affect their prominence. This knowledge could be valuable for building algorithms for automatic extraction of image prominent colours, which have many different application values, e.g. they can be used for image categorisation, colour-based image retrieval or colour schemes extraction.

In the past, several techniques and methods for automatic colour extraction were presented, however, so far, little attention has been paid to develop an algorithm for extracting most prominent colours. Most techniques focus on extracting representative colours, which are not always the ones that are most prominent. Techniques for extracting colours from an image can be classified into three main categories: clustering-based methods, histogram-based methods and data-driven methods. Clustering-based algorithms categorised or grouped colours in the image - values of image pixels, based on specific criteria and use the centroid or mean value of each cluster as representative colours. A well-known clusteringbased algorithm is k-mean, which goal is to group colours into a k number of clusters in order to minimise the within-cluster sum of squares - squared distances between each colour and the mean value of their corresponding cluster. While the k-mean algorithm assigns each pixel value to exactly one cluster, i.e. hard clustering, in fuzzy or c-means clustering, colours can belong to more than one cluster, i.e. soft clustering. Despite the popularity and convenience of the clustering-based algorithm, Lin and Hanrahan found out that people tend to select different colours in comparison to colours extracted with popular clustering methods, such as k-means and c-means (Lin, 2013). Few researchers have presented methods for extracting image representative colours based on histogram analysis. Based on the observation that people tend to select colours from distinctive regions of the image, Delon et al. developed an algorithm that finds peaks of one-dimensional histograms of hue, saturation and lightness, which according to their opinion, correspond well to spatial regions (Delon et al, 2007). Colours that represent histogram peaks can be used directly or as initial seeds for the clustering methods as mentioned above. Morse et al. proposed a similar histogram-based method, which also allows users to specify a maximum number of extracted colours and a minimum distance between them. The method strives to find peaks in the hue 
histogram, which is weighted according to saturation and spatial coherence of each pixel in the image (Morse et al, 2007). With the increase in computational power that has enabled the recent breakthroughs in machine learning, the data-driven model has gained considerable research interest. Lin and Hanrahan presented a regression model for extracting colour schemes from images (Lin et al, 2013). The model is trained on user-generated colour schemes and uses more than a hundred features. In contrast to the algorithms that only consider the colour information when extracting colours, Liu and Luo presented a method that also considers users' emotion and feeling about the image (Liu et al, 2016).

As discussed above, no previous study has focused on developing an algorithm for extracting image prominent colours. In order to be able to develop such an algorithm, extensive knowledge about the perception of image prominent colours and its influencing factors is needed. The application presented in this paper provides a simple and efficient way to create a database of image prominent colour, which is a basis for further studies.

\section{APPLICATION OVERVIEW}

This section starts with an overview of the application structure and then continues with a more detail description of application's settings and functionality.

\subsection{Architecture overview}

The application is developed based on a model-view-controller (MVC) architectural pattern, using popular Javascript library React (Reactjs, 2018). React was built by Facebook and is mainly used for developing single-page or mobile applications (progressive web applications). The main advantage of using React library lies in one-way data flow and virtual Document Object Model (DOM). The latter has a massive impact on the performance since the real DOM is only rendered when needed, while the oneway data flow allows robust and less-error-prone development. The overall structure of the application is shown in Figure 1. The processing in control and view part of the application is carried out directly in a client-side environment (browser). Consequently, each stage of application is loaded instantly and responds quickly to user interactions. To process data on the server-side and save results in a database, the PHP programming language and MYSQL database is used, respectively. The interface of the application was designed using Material Design design language and responsive web design (RWD) approach, which allows different page rendering on different devices and screen sizes, in order to optimise user experience. The main stage of the application, as it renders on desktop and tablet devices, is shown in Figure 2. At the moment, the application is not supported on devices with a width lower than 768px.

\subsection{Input parameters}

The application provides a number of settings that control its functionality and can be set through URL GET parameters. Bellows are the input parameters with their supported values and their corresponding data types:

- num_img (integer) sets the number of images that will be used in an experiment. If this parameter is omitted all images in the set will be used.

- colour_mode (string: hues | cc24 | custom) sets the colour selection mode (see section 2.3). By default cc24 (ColorChecker Classic target) mode is used.

- $\quad \min$ (int) sets the minimum number of colours that need to be selected by the observer. The default value is 3 .

- $\quad \max$ (int) sets the maximum number of colours that are allowed to be selected. The default value is 7 .

- $\quad$ shuffle (boolean) controls the order of presented colour patches. If set to true, the patches will be shuffled before being shown to the observer. Otherwise, they will be displayed as defined in a file. This parameter is active only in ColorChecker Classic and Custom colour modes.

- sortable (boolean) controls if selected colours can be sorted or not. The default value is true, which allows the observer to sort selected colours by drag and drop gesture. 


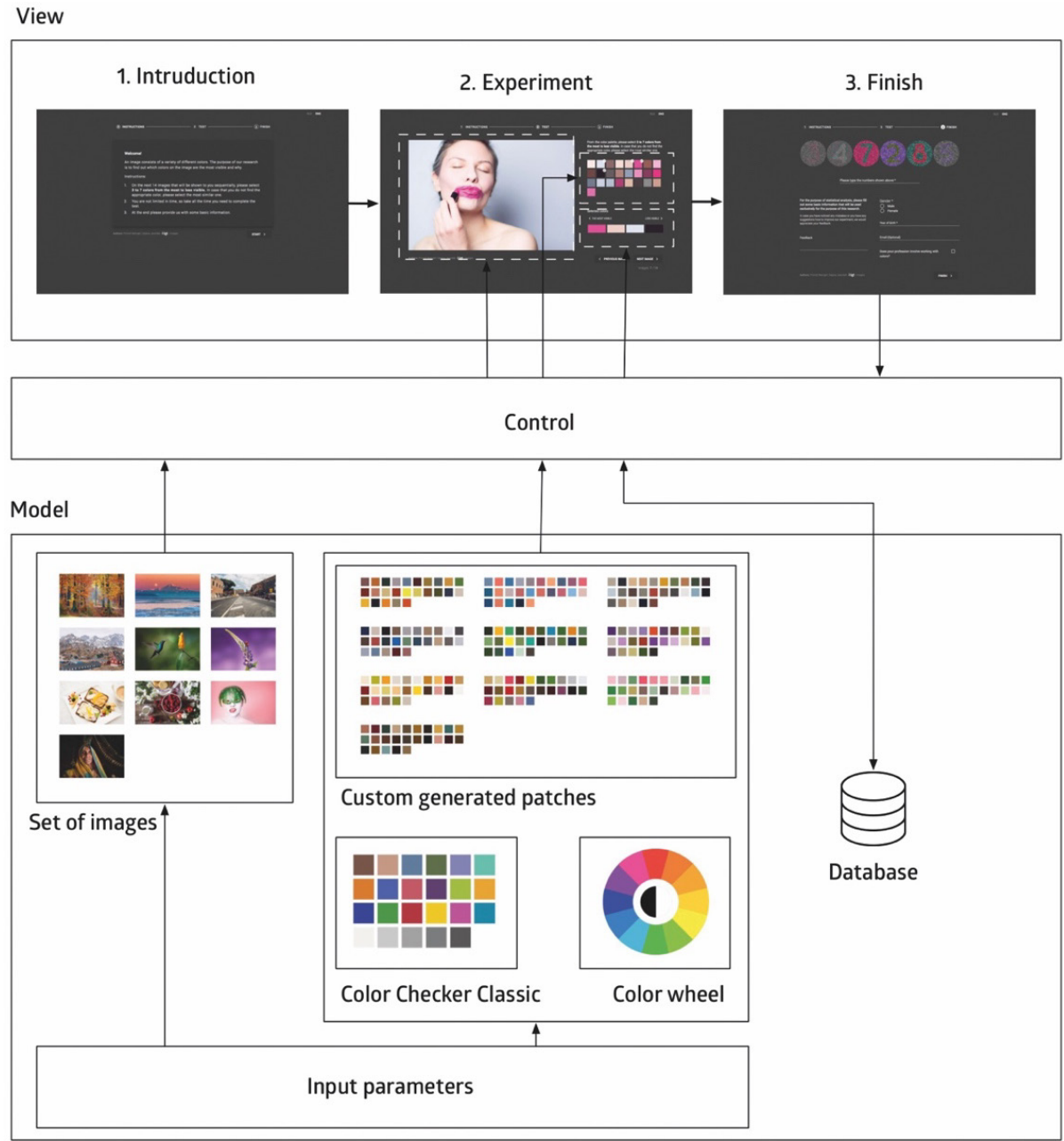

Figure 1: MVC architectural pattern of application.

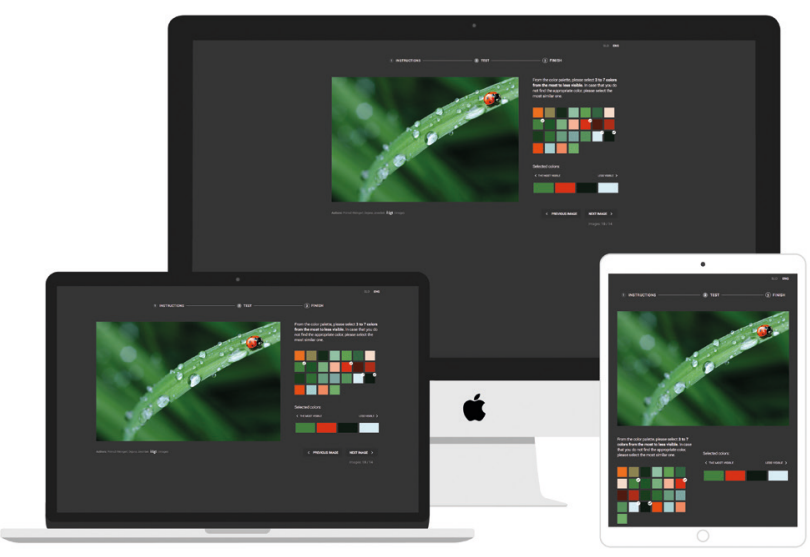

Figure 2: Design of the application interface, as it renders on three different screen sizes.

\subsection{Colour selection modes}

An 8-bit RGB image can theoretically contain more than 16.7 millions colours. If the observer could select any colour from the image, we would obtain very precise sets of prominent colours. However, because some colours on the image can be quite similar and therefore indistinguishable for most observers, the variation between the observers - interobserver variability - will be considerable. Such results would be challenging to statistically analyse and provide little opportunity to make a further generalisation about the perception of prominent colours. In addition, selecting from the large set of colour patches can be frustrating for the observer and could decrease the usability of the application. Our application allows 
the research administrator to balance between data precision and usability of the application, by supporting three different modes for selecting the colours. All three modes can be seen in Figure 3 and are explained below.

1. Prominent hues: this mode allows the observer to select only from the 12 basic hues: red, red-orange, orange, orange-yellow, yellow, yellow-green, green, cyan, cyan-blue, blue, purple and magenta. In addition, the observer can also select a black and white colour.

2. ColorChecker Classic: the observer can select from 24 patches of the ColorChecker Classic target. This target contains natural, chromatic, primary and grayscale colours, which represent natural objects, such as human skin, foliage and blue sky.

3. Custom: this mode allows the research administrator to define a custom set of colours for each image. The colours can be generated by using common extraction algorithms, such as K-means or K-fold. To import colours into the application, the colours must be defined inside the JSON file as a multidimensional array of objects, containing CIELAB and SRGB values.
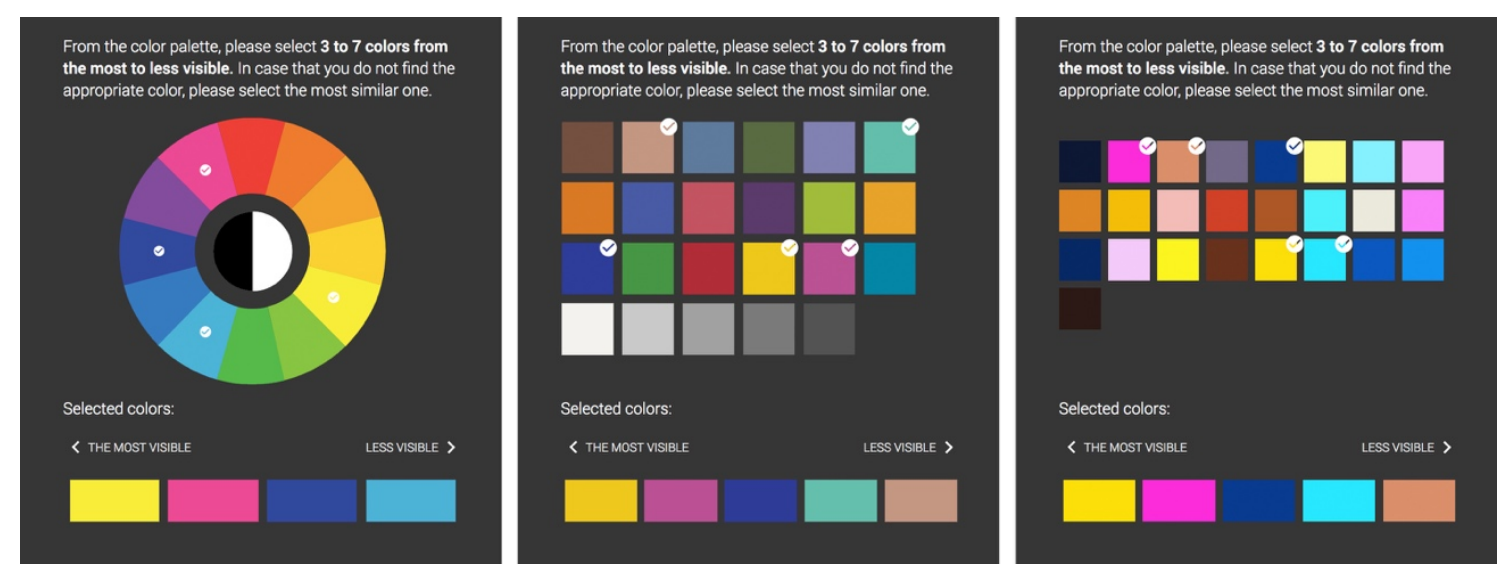

Figure 3: Colour selection modes of the application: Prominent hues (left), ColorChecker Classic patches (middle), Custom defined patches (right).

\section{APPLICATION USAGE}

Although the main purpose of our application is to gather prominent colours of images based on the observer's opinion, the application could also be used for other psychophysical experiments or purposes, e.g. if we want to find out which colours are harmonious on the image, or to get a colour scheme for a particular image without special restrictions. In the following, the detail description of how to use the application for collecting image prominent colours is given based on our case study.

\subsection{Defining input parameters and images}

In the presented case study we used 4 sets of 12 images. Participants in the study were asked to select 3 to 7 colours for each image, from the most to less prominent. They could select from 25 colours (custom colour selection mode), that were extracted from images in advance using the k-mean algorithm.

\subsection{Collecting data}

The process of collecting image prominent colours consists of three main parts: introduction, colour selection task and observer information task (Figure 4). In the first step, the introductory text, which explains the main task and the criteria that need to be taken into account when selecting colours, is shown. In the next step, images are sequentially displayed in pseudorandom order. For each image, the observer has to select colours that are most prominent in the image. If colour sorting is enabled (see section 2.2), the observer can also sort colours, based on their prominence, with drag and drop gesture. Otherwise, the colours are ordered based on their initial selection - from most prominent in the far right to the least prominent on the far left. In our case, the colour sorting was disabled. When the observer is satisfied with the selected colours and their order, he or she can continue to the next image. After all images have been displayed, the observer is redirected to the last step of the process, where he or she is asked to provide some personal information. In addition, the observer has to type numbers that 
are visible in Ishihara colour test images, so that potential colour vision deficiencies of the observers can be determined when analysing results. At the end of the process, all collected data about selected colours and the observer are written to the database and observer is prompt to repeat the process with another set of images.

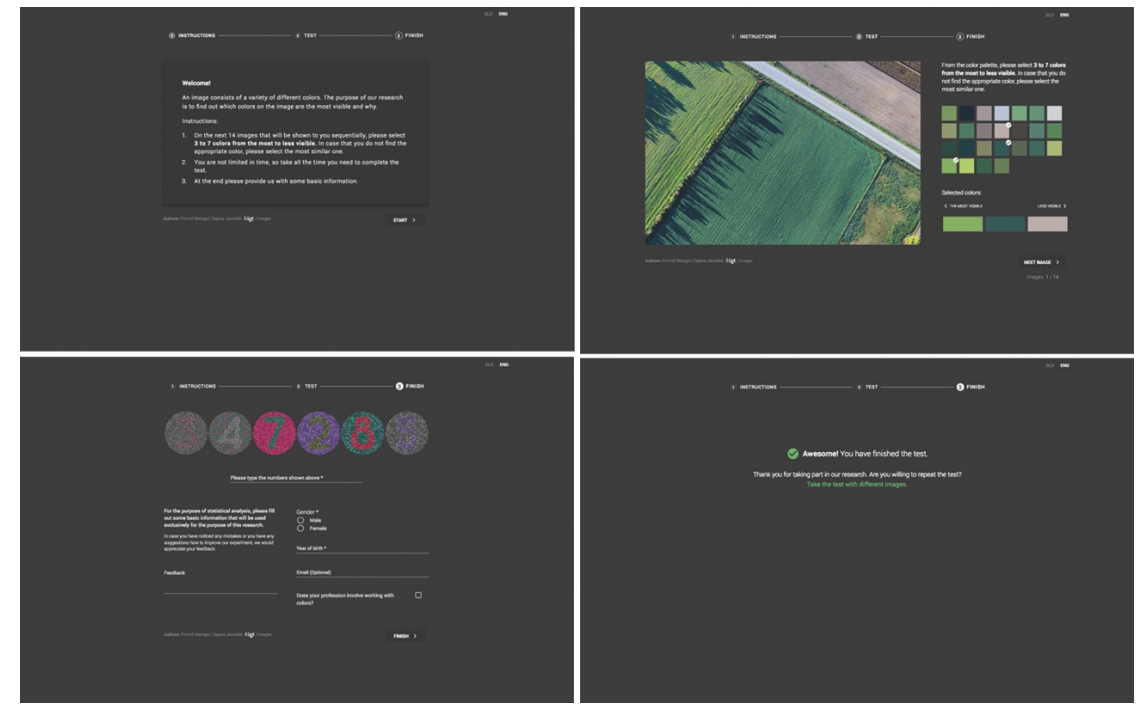

Figure 4: Three main steps for collecting data - introduction (top left), colour selection task (top right) and observer information task (bottom left) - and final notice at the end of the process (bottom right).

\subsection{Viewing, editing and visualising data}

For instant access to the results, while the research is still in process, we developed an interface, which allows the administrator an option to view, edit and visualise the collected data. In the top-left corner of the interface, the image and colours that were available for selection are displayed, just as it has been shown to the observers. The selected colours and personal data for each observer are displayed in a table at the right side of the interface. Each row represents data for each observer and can be edited or deleted if necessary. At the bottom, selected colours are visualised in a user-friendly manner. Each row represents the frequency of selecting a particular colour, whereas columns represent selected colours for each observer. Thus, the agreement between observers (interobserver reliability) and dispersion of selected colours can be easily discerned. In addition, the research administrator can export data in CSV or XLXS format for further analysis. The screenshot of the interface is shown in Figure 5.

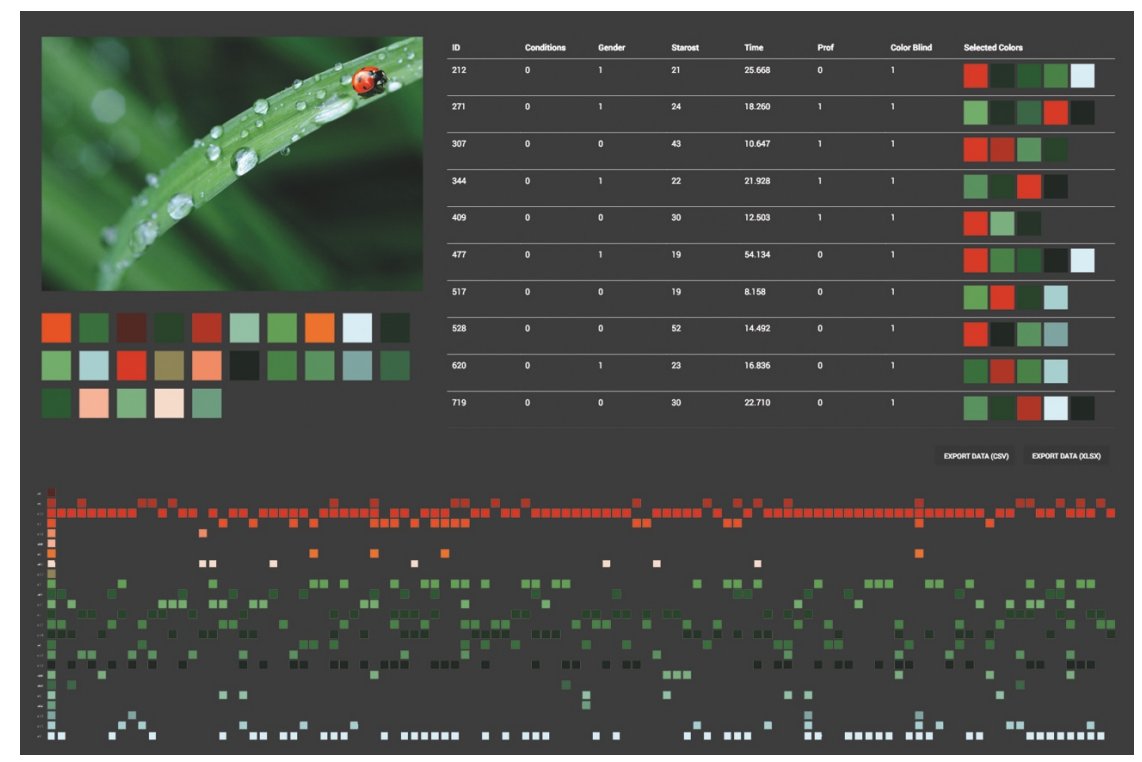

Figure 5: The interface for viewing, editing and visualising collected data 


\section{CONCLUSION}

In this paper, the application for collecting image prominent colours, based on the observer's opinion has been presented. The application can also be used for conducting other psychophysical experiments, where observers have to select colours from the image according to specific criteria. The main advantages of our application are simplicity to use and effectiveness since colours are being selected interactively and data are collected automatically without any assistance from a research administrator.

\section{REFERENCES}

[1] Delon, J., Desolneux, A., Lisani, J. L., Petro, A. B.: "Automatic color palette", Inverse Problems and Imaging, 2(2), 706-709, 2005. doi: 10.1109/ICIP.2005.1530153

[2] Kopacz, J.: "Enhancing design using color", In book: Colour Design, 2012. doi: 10.1533/9780857095534.3.336

[3] Liu, D., Jiang, Y. X., Pei, M., and Liu, S. G.: "Emotional image color transfer via deep learning", Pattern Recognition Letters, 110, 16-22, 2018. doi: 10.1016/j.patrec.2018.03.015

[4] Lin, S., Hanrahan, P.: "Modeling how people extract color themes from images", Proceedings of the SIGCHI Conference on Human Factors in Computing Systems, 2013. doi: 10.1145/2470654.2466424

[5] Liu, S., Luo, H.: "Hierarchical emotional color theme extraction", Color Research \& Application, 41(5), 513-522, 2016. doi: 10.1002/col.21988

[6] Morse, B. S., Thornton, D., Xia, Q., Uibel, J.: "Image-Based Color Schemes", Image Processing, 2007. ICIP 2007. IEEE International Conference, 2007. doi: 10.1109/ICIP.2007.4379355

[7] Ou, L. C., Luo, M. R., Woodcock, A., Wright, A.: "A study of colour emotion and colour preference Part I: Colour emotions for single colours", Color Research and Application, 29(3), 232-240, 2004. doi: $10.1002 /$ col.20010

[8] React, A JavaScript library for building user interfaces, Reactjs, URL: https://reactjs.org/ (last request: 2018-09-18).

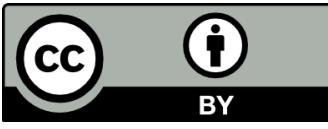

(C) 2018 Authors. Published by the University of Novi Sad, Faculty of Technical Sciences, Department of Graphic Engineering and Design. This article is an open access article distributed under the terms and conditions of the Creative Commons Attribution license 3.0 Serbia (http://creativecommons.org/licenses/by/3.0/rs/). 